\title{
Erratum to: A combination of the telomerase inhibitor, BIBR1532, and paclitaxel synergistically inhibit cell proliferation in breast cancer cell lines
}

$\mathrm{Yi} \mathrm{Shi}^{1} \cdot \operatorname{Lin} \mathrm{Sun}^{1} \cdot \mathrm{Ge} \mathrm{Chen}^{1,2} \cdot$ Dongyan Zheng ${ }^{1} \cdot \mathrm{Li} \mathrm{Li}^{1} \cdot$ Wanguo Wei ${ }^{1,2}$

Published online: 24 January 2017

(C) Springer International Publishing Switzerland 2017

Erratum to: Targ Oncol (2015) 10:565-573

DOI 10.1007/s11523-015-0364-y

A funding statement was omitted from the original publication of this article.

Page 572: The Acknowledgements, which previously read: "This work was supported by the National Natural Science Foundation of China (21202100) and the Strategic Priority Research Program of the Chinese Academy of Sciences (XDA01040302)."

should read:

"This work was supported by the Chinese Ministry of Science and Technology (2013CB967402, granted to Li Li), the National Natural Science Foundation of China (21202100) and the Strategic Priority Research Program of the Chinese Academy of Sciences (XDA01040302).”

The online version of the original article can be found at http://dx.doi. org/10.1007/s11523-015-0364-y.

$\mathrm{Li} \mathrm{Li}$

lil@sari.ac.cn

$\triangle$ Wanguo Wei

weiwg@sari.ac.cn

1 Shanghai Advanced Research Institute, Chinese Academy of Sciences, 99 Haike Road, Zhangjiang Hi-Tech Park,

Pudong, Shanghai 201210, China

2 School of Life Science and Technology, ShanghaiTech University, 319 Yueyang Road, Shanghai 200031, China 